\title{
Technical note: An apparatus for catheterization of the lateral brain ventricle in Holstein cows
}

\author{
B. Kuhla, ${ }^{1}$ O. Bellmann, and C. C. Metges \\ Leibniz Institute for Farm Animal Biology (FBN), Wilhelm-Stahl-Allee 2, 18196 Dummerstorf, Germany
}

\begin{abstract}
A stereotaxic apparatus for access to the lateral brain ventricle of Holstein cows was constructed. This apparatus permits topographic orientation on the head of an anesthetized cow that has been placed in right lateral recumbency. After assessing the XY-plane in parallel to the frontal bone, correct coordinates in reference to the skullcap were adjusted. The lateral brain ventricles were accessed by drilling holes perpendicular to the XY-plane. The subsequent surgical implant of a tailormade cannula guide enabled the repeatable placement of a catheter. This permanent catheter allowed the frequent withdrawal of cerebrospinal fluid from and injections into cerebral ventricles of nonanesthetized dairy cows. This method is critical for elucidating the role of central hormones, metabolites, and electrolytes in the regulation of diverse physiological processes and for the study of neuropathological disorders in cows.

Key words: stereotaxic apparatus, cannulation, dairy cow, cerebrospinal fluid
\end{abstract}

Stereotaxic techniques facilitate the investigation of neurophysiological functions. Several stereotaxic procedures for accessing defined brain areas were established for calves many years ago (Manchanda et al., 1971; Stewart and Bailey, 1973; Hedlund et al., 1977). While the calf is in standing position or placed in sternum recumbency, application of stereotaxic tools alleviates the adjustment to the plane of the frontal bone. Surgical implantation of cannula-guided catheters into brain ventricles of calves enables serial sampling of cerebrospinal fluid or injection studies (Barlow et al., 1978; Leshin et al., 1990; Ames et al., 1995). Because these stereotaxic instruments were originally constructed for the head size of calves, their use for surgeries on the much larger heads of mature cattle is inappropriate. Furthermore, the more pronounced poll of cows compared with that of calves, differences in the topography of the brain in relation to the skullcap (Barlow et al.,

Received May 12, 2010.

Accepted August 20, 2010.

${ }^{1}$ Corresponding author: b.kuhla@fbn-dummerstorf.de
1978), as well as broad sex and breed differences present further problems for a simple enlargement of calfspecific stereotaxic instruments. Studies on Jersey cows dispensed with the use of exact stereotaxic coordinates and a permanent catheter by implanting a large area septum in the frontal bone (Cox and Littledike, 1978). X-ray (Gazal et al., 1998; Thomas et al., 1999) and ultrasound-aided (Yoshioka et al., 2001) techniques for visualizing the third ventricle of cows with subsequent catheterization have been described. Both methods require expensive diagnostic apparatus or a large hole in the frontal bone for ultrasonic orientation (Yoshioka et al., 2001). However, anatomical landmarks and stereotaxic coordinates to access the lateral ventricle of mature dairy cows are lacking, calling for the development of an appropriate stereotaxic instrument. Here, we describe a new apparatus used for the catheterization of the lateral brain ventricle in Holstein cows.

All experimental procedures were conducted in accordance to the guidelines for the care and use of animals as experimental subjects of the State Government in Mecklenburg-West Pomerania (Germany). These procedures were officially approved (Registration No. LALLF M-V/TSD/7221.3-1.2-047/07).

The apparatus is composed of 3 plexiglass [poly (methyl methacrylate)] components, the baseboard, the drill guide plate, and the bulbus bar (a, b, and c in Figure 1) that allow orientation of the head due to their transparency. The 8-mm-thick baseboard is $57 \mathrm{~cm} \mathrm{long,}$ $20 \mathrm{~cm}$ wide in the upper third, and $10 \mathrm{~cm}$ wide in the lower two-thirds, illustrating the XY-plane in front of the forehead. To affix it to the posterior part of the head, a clamp composed of $0.8-\mathrm{mm}$ stainless steel may be moved perpendicular to the baseboard transversal plane along its 7-cm longitudinal slot (no. 4, Figure 1B). The $90^{\circ}$ bent head of the clamp, which is in parallel to the baseboard plane, features a semicircle shape to grip behind the poll. Securing the clamp position via the fixing screw (no. 5, Figure 1B) tightly connects the baseboard at the poll of the cow. At the lower twothirds of the baseboard, a $0.8-\mathrm{mm}$ stainless-steel shoe (no. 1, Figure 1A) is screwed at the backside of the baseboard. The shoe sole is $7 \mathrm{~cm}$ wide and each check $(10 \times 10 \mathrm{~cm})$ is bent $10^{\circ}$ apart. The 2 checks of the shoe 

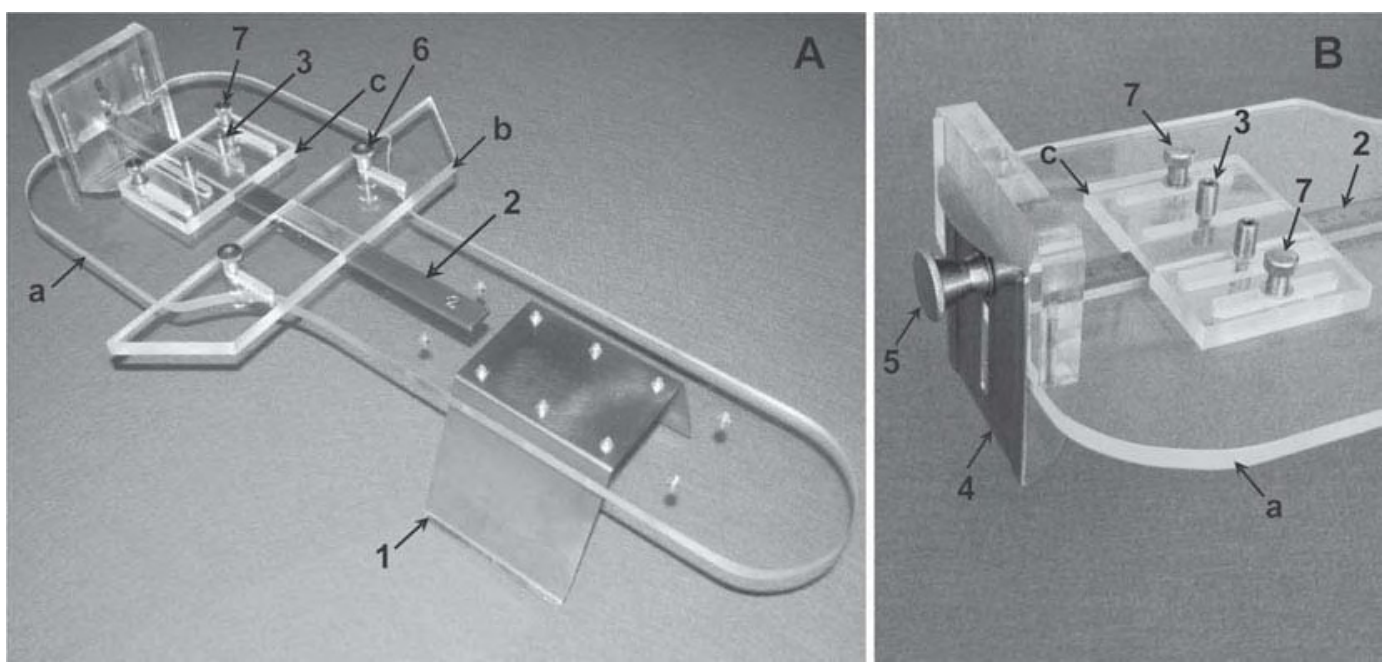

Figure 1. Stereotaxic apparatus for mature cows: front view (A) and rear view (B). Plexiglas components: baseboard (a), bulbus bar (b), drill guide plate (c), stainless steel components: shoe (1), 30-cm calibrated straightedge (2), drill sleeve (3), clamp (4), clamp fixing screw (5), bulbus bar fixing screw (6), and drill guide plate fixing screw (7).

may be tightly squeezed on the cow's nasal bone, thus keeping the baseboard secure in the transversal plane. With a belt (Figure 2A) attached through the jaw, the baseboard can be toggled to the anterior head. In the midline of the baseboard, a $30-\mathrm{cm}$, calibrated straightedge (accurate to $1 \mathrm{~mm}$ ) composed of stainless steel $(4 \times 18 \mathrm{~mm})$ is screwed on top (no. 2, Figure 1). Null is set at the upper edge of the baseboard and crosses the plane of the clamp. Furthermore, $21 \mathrm{~cm}$ from the upper end of the baseboard and $6 \mathrm{~cm}$ laterally from each side of the midline, 2 threads with fixing screws are arrayed (no. 6, Figure 1A). On these screws, the bulbus bar (b, Figure 1A) may be slid and toggled in a range of its $4-\mathrm{cm}$ longitudinal slots in antero-posterior direction. The bulbus bar is trapezoidal (29 to $20 \times$ $6 \mathrm{~cm}$ ) and is countersunk on its back side, enabling sliding on the straightedge. In a similar way, the rectangular drill guide plate $(7 \times 10 \mathrm{~cm})$ can be moved, due to a countersunk slot at its back side, along the straightedge in an antero-posterior direction. Briefly, $9.5 \mathrm{~cm}$ from upper end and each $4 \mathrm{~cm}$ lateral from the midline, 2 threads are placed on the baseboard. At the latter, fixing screws may secure the drill guide plate at any position of its 5-cm longitudinal slots (no. 7, Figure 1). To ensure drilling perpendicular to the transversal plan, a 3-cm-high stainless drill sleeve $(3.6 \mathrm{~mm}$ i.d.; 8 $\mathrm{mm}$ o.d.) is embedded on each side $1.4 \mathrm{~cm}$ lateral from the midline of the drill guide plate (no. 3, Figure 1). Because of its inner diameter, the drill sleeve allows only a surgical drill of $3.5 \mathrm{~mm}$. A thin transversal line between the drill sleeves is sketched on the drill guide plate, enabling a reading to be taken at the straightedge. The area that is covered by the drill sleeves is routed in the baseboard, yielding each a 6.5 -cm longitudinal slot; this enables the passage of the drill to the frontal bone.

Landmarks and adjustments at the stereotaxic apparatus for accessing the lateral brain ventricle were initially determined on 6 carcass heads and later confirmed in surgeries. At first, the steel clamp (no. 4, Figure 1B) and then the belt (Figure 2A) were toggled at the posterior and anterior head, respectively, to affix the baseboard. The longer site of the bulbus bar was secured in a position where it displays the transverse line between the caudal margins of the cow's eye orbits (Figure 2B). The measured value at the calibrated straightedge was then divided by 2 (Figure 2B). The calculated result provides the value that was adjusted on the straightedge with the transversal line scratched on the drill guide plate. The drill sleeve on the drill guide plate, which is arrayed $1.4 \mathrm{~cm}$ lateral from the midline, determined the position for drilling (Figure 2B). Because of its size, the stereotaxic apparatus could be used in surgeries of anesthetized cows placed either on sternal or lateral recumbency.

To guide the catheter, a 55-mm-long, 11-gauge syringe needle was constructed (Figure 3A). This length is sufficient to penetrate through the $2-\mathrm{cm}$ skull and about $3.5 \mathrm{~cm}$ into the cerebral cortex. To enable attachment to the bone, a 3 -hole titanium plate $(38 \times 10 \times 3 \mathrm{~mm}$; B. Braun Melsungen AG, Melsungen, Germany) was soldered in its middle hole to the base of the Luer-lock of the cannula. The remaining 2 holes of the titanium plate (Figure 3B) served as anchor points for 2 selfthreading screws (Figure 3C). The female Luer-lock of the cannula can be capped with a septum-equipped male plug (Figure 3A). A protection sleeve made of 

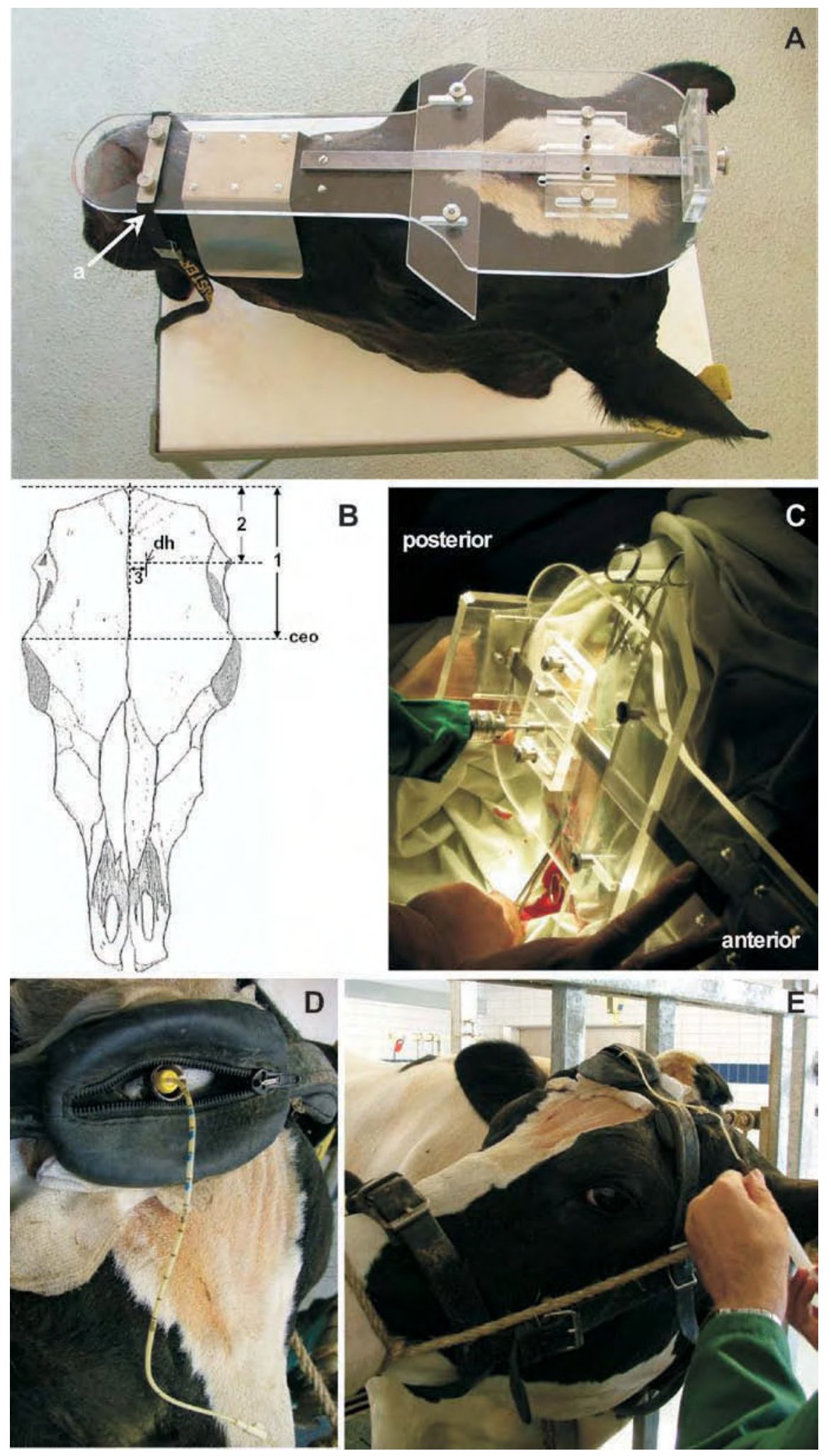

Figure 2. Stereotaxic apparatus secured with a belt (a) to a carcass head (A). Frontal diagram of a cow skull showing landmarks and measurements for drilling to access the lateral brain ventricle (B): transverse line between the caudal margins of the cow's eye orbits (ceo), distance between the caudal poll margin and the transverse line between the caudal eye orbits (1), distance (1) divided by $2(2), 1.4 \mathrm{~cm}$ lateral from the midline (3), place of the drill hole (dh). Stereotaxic apparatus in use for drilling a hole into the frontal bone (C). Halter with catheter bag (D) and forehead strap (E). Color version available in the online PDF. 

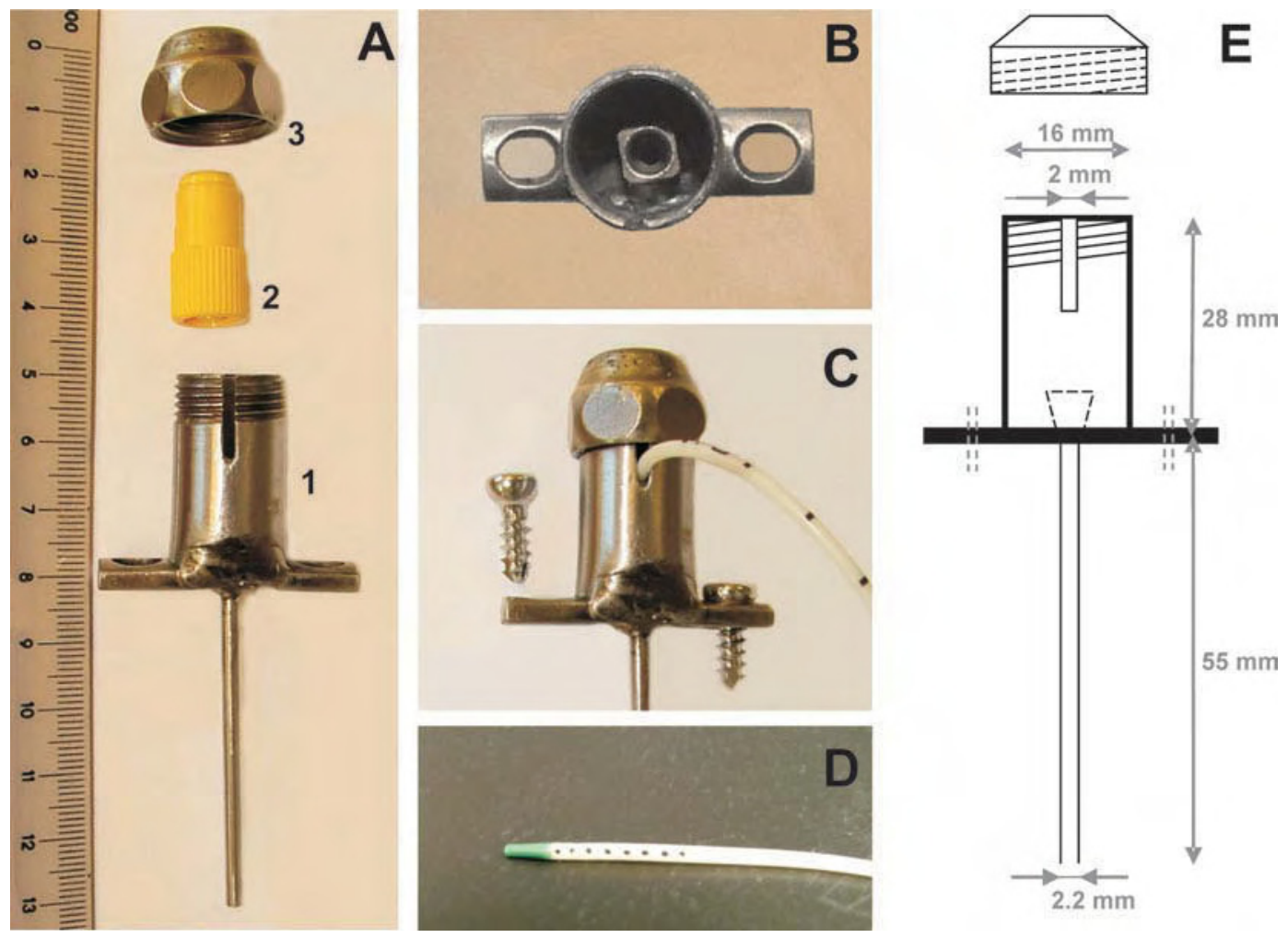

Figure 3. Cannula guide (1), septum (2), and thread lid (3) with male plug (A); view on top of the cannula guide without septum (B); protection sleeve closed by the thread lid: the catheter goes through the side slot; 2 self-threading screws for anchorage at the frontal bone (C); catheter tip with additional 0.7-mm-diameter holes on side (D); line diagram of the cannula guide (E). Color version available in the online PDF.

stainless-steel tube (diameter: $16 \times 28 \mathrm{~mm}$ ) with female thread was soldered on the titanium plate surrounding the Luer-lock with the septum-equipped male plug. A lateral $12-\times 2$-mm slot was sawed perpendicular to the thread for the outlet of the catheter when the steel male plug closes the protection sleeve (Figure 3C). At the tip of the catheter tubing (CertofixMono, 14-gauge, $30 \mathrm{~cm}$; B. Braun Melsungen AG), 6 to 8 additional 0.7-mm-diameter holes were drilled on the side over a distance of $1.5 \mathrm{~cm}$ (Figure 3D) to enable withdrawal of cerebrospinal fluid (CSF) when some of the holes are clogged.

Six Holstein cows, weighing between 480 and $680 \mathrm{~kg}$ and adapted to tiestalls with individual feed bins, had feed withheld for $12 \mathrm{~h}$ before surgery. The forehead was shaven and a catheter was placed into the jugular vein. Animals were sedated with xylazine $(0.1 \mathrm{mg} / \mathrm{kg}$; Riemser Arzneimittel AG, Insel Riems, Germany) and ketamine (1 mg/kg; Serumwerk Bernburg AG, Bernburg, Germany) via the jugular catheter (CertofixMono, 14-gauge, $30 \mathrm{~cm}$; B. Braun Melsungen AG) and placed in lateral recumbency for surgery. The head was prepared for sterile surgery and a triangular incision (approximately $5 \times 5 \mathrm{~cm}$ ) was made to expose the fron- tal bone in the middle of the distance between caudal eye orbit and caudal margin of the poll (position dh, Figure 2B). The stereotaxic apparatus was affixed at the head and the adjustments for accessing the lateral brain ventricle via the drill sleeve were performed as described above. A 3.5-mm-diameter hole was drilled in the frontal bone (Figure 2A) through which the cannula guide was lowered and thereby punctured the meninges. Next, the catheter with guide wire was pressed through the septum plug far enough for the tip to reach the expected depth of the lateral ventricle $(\sim 6 \mathrm{~cm})$. The catheter was lowered through the cannula guide and the septum was tightened to the Luer-lock of the cannula. The subsequent removal of the guide wire was generally accompanied by outflow of CSF. Otherwise, the catheter was pushed in further or pulled out until outflow of CSF was observed. The Luer-lock outlet of the catheter was closed by a stopcock. Then, the cannula guide at its titanium plate was secured via the 2 holes on the frontal bone with self-threading screws (4.5 $\times 16 \mathrm{~mm}$ diameter; B. Braun Melsungen AG; Figure 3C) using an Allen key. The catheter was pushed in the side slot of the protection sleeve and the thread lid was screwed on the catheter guide (Figure $3 \mathrm{C}$ ). After the 
skin was sutured, the scar was covered with ImmuStim lotion (Almapharm, Kempten, Germany) and covered with gauze. For protective storage of the catheter and stopcock, a catheter bag was placed on the protection sleeve of the cannula guide (Figure 2D). The bag was attached to a leather forehead strap, which ranged between the eye orbits and the ears, and mounted with buckles to the halter (Figure 2E). The whole procedure took between 50 and $70 \mathrm{~min}$, and the cow usually recovered from anesthesia within $12 \mathrm{~h}$.

After recovery, animals were housed in tiestalls and received analgesia (metapyrine, 50mg $/ \mathrm{kg}$; Medistar GmbH, Hannover, Germany) and antibiotics (sulfadimidin sodium, $22 \mathrm{mg} / \mathrm{kg}$, and trimethoprim, $4 \mathrm{mg} /$ $\mathrm{kg}$; Medistar $\mathrm{GmbH}$ ) for 3 d after surgery. During this time, the integrity of the catheter was verified twice daily by withdrawal of 0.5 to $1 \mathrm{~mL}$ (in some cases of 2 $\mathrm{mL}$ ) of CSF. If CSF was not obtainable, the patency of the catheter was reconstituted either by pressing CSF located in the catheter with an air-filled syringe or by poking with the guide wire. Between samplings, the guide wire was not placed in the catheter. The integrity of the catheters lasted between 5 and $14 \mathrm{~d}$. Thereafter, clogging or abrasion of the catheter, or, in one case, deflexion of the cannula guide, explained the lost patency of the catheter. In one case, the catheter tube was replaced on a tranquilized (xylazine; $0.02 \mathrm{mg} / \mathrm{kg}$ ) cow 5 $\mathrm{d}$ after surgery. Full feed intake was generally resumed 2 to 5 d postsurgery. After $5 \mathrm{~d}$, sutures were removed.

In conclusion, we describe a stereotaxic apparatus for the catheterization of the first or second brain ventricle of mature Holstein cows. The technique can be seen as an extension to the methods developed earlier for calves and should assist in the safe conduct of neurophysiological studies on cows. The technique minimizes the danger of infection by using only small drill holes, it allows the application on laterally recumbent animals without using a special cart for sternal placement, it requires no expensive visualization techniques as needed for third ventricle cannulation (Gazal et al., 1998; Thomas et al., 1999; Yoshioka et al., 2001), and it uses an improved catheter.

\section{ACKNOWLEDGMENTS}

This study was financed by the core budget of the Leibniz Institute for Farm Animal Biology (FBN, Dummerstorf, Germany). We thank FBN staff Ernst Paetow and Jürgen Humboldt for their excellent technical support, Claudia Arlt for graphic design, Elke Albrecht for taking pictures of the stereotaxic apparatus, and the staff at the FBN animal facility and the 'Tiertechnikum.' Bob Christopherson (University of Alberta, Edmonton, AB, Canada) is gratefully acknowledged for linguistic refinements.

\section{REFERENCES}

Ames, N. K., H. A. Tucker, L. T. Chapin, and P. J. Gaynor. 1995. Cannulation of a lateral ventricle in the brain of Holstein calves. Am. J. Vet. Res. 56:199-202.

Barlow, R. M., R. C. Dow, P. Harris, B. Mitchell, and H. R. A Townsend. 1978. Surgical techniques for the serial sampling of cerebrospinal fluid and for electrocorticography in cattle. Res. Vet. Sci. 24:358-365.

Cox, P., and E. T. Littledike. 1978. Techniques for sampling ventricular and cisternal cerebrospinal fluid from unanesthetized cattle. Lab. Anim. Sci. 28:465-469.

Gazal, O. S., L. S. Leshin, R. L. Stanko, M. G. Thomas, D. H. Keisler, L. L. Anderson, and G. L. Williams. 1998. Gonadotropin-releasing hormone secretion into third-ventricle cerebrospinal fluid of cattle: correspondence with the tonic and surge release of luteinizing hormone and its tonic inhibition by suckling and neuropeptide Y. Biol. Reprod. 59:676-683.

Hedlund, L., M. M. Lishko, and H. E. Garner. 1977. Cerebral ventricle cannulation in the calf. J. Dairy Sci. 60:997-1000.

Leshin, L. S., L. A. Rund, F. N. Thompson, M. B. Mahaffey, W. J. Chang, D. J. Byerley, and T. E. Kiser. 1990. Serum prolactin and growth hormone responses to naloxone and intracerebral ventricle morphine administration in heifers. J. Anim. Sci. 68:1656-1665.

Manchanda, S. K., K. Singh, and B. K. Soni. 1971. Stereotaxic apparatus for the buffalo (Bubalus bubalis) brain. Physiol. Behav. 6:727-728

Stewart, R. E., and E. M. Bailey Jr.. 1973. Hypothalamic temperature regulation in cattle. Trans. ASAE 16:334-339.

Thomas, M. G., O. S. Gazal, G. L. Williams, R. L. Stanko, and D. H. Keisler. 1999. Injection of neuropeptide $\mathrm{Y}$ into the third cerebroventricle differentially influences pituitary secretion of luteinizing hormone and growth hormone in ovariectomized cows. Domest. Anim. Endocrinol. 16:159-169.

Yoshioka, K., C. Suzuki, S. Arai, S. Iwamura, and H. Hirose. 2001. Gonadotropin-releasing hormone in third ventricular cerebrospinal fluid of the heifer during the estrous cycle. Biol. Reprod. 64:563-570. 